\title{
Some Properties of Kantorovich-Stancu-Type Generalization of Szász Operators including Brenke-Type Polynomials via Power Series Summability Method
}

\author{
Naim Latif Braha, ${ }^{1,2}$ Toufik Mansour, ${ }^{3}$ and Mohammad Mursaleen $\mathbb{1 D}^{4,5,6}$ \\ ${ }^{1}$ Ilirias Research Institute, rr-Janina, No-2, Ferizaj 70000, Kosovo \\ ${ }^{2}$ Department of Mathematics and Computer Sciences, University of Prishtina, Avenue Mother Teresa, No-5, Prishtine 10000, Kosovo \\ ${ }^{3}$ Department of Mathematics, University of Haifa, 3498838 Haifa, Israel \\ ${ }^{4}$ Department of Medical Research, China Medical University Hospital, China Medical University (Taiwan), Taichung, Taiwan \\ ${ }^{5}$ Department of Mathematics, Aligarh Muslim University, Aligarh 202002, India \\ ${ }^{6}$ Department of Computer Science and Information Engineering, Asia University, Taichung, Taiwan
}

Correspondence should be addressed to Mohammad Mursaleen; mursaleenm@gmail.com

Received 6 May 2020; Accepted 12 June 2020; Published 14 August 2020

Academic Editor: Lars E. Persson

Copyright (c) 2020 Naim Latif Braha et al. This is an open access article distributed under the Creative Commons Attribution License, which permits unrestricted use, distribution, and reproduction in any medium, provided the original work is properly cited.

In this paper, we study the Kantorovich-Stancu-type generalization of Szász-Mirakyan operators including Brenke-type polynomials and prove a Korovkin-type theorem via the $T$-statistical convergence and power series summability method. Moreover, we determine the rate of the convergence. Furthermore, we establish the Voronovskaya- and Grüss-Voronovskayatype theorems for $T$-statistical convergence.

\section{Introduction and Preliminaries}

Let $\mathscr{K} \subseteq \mathbb{N}$ (set of natural numbers) and $\mathscr{K}_{m}=\{i \leq m: i \epsilon$ $\mathscr{K}\}$. Then, the natural density or we can say asymptotic density of $\mathscr{K}$ is defined by $\sigma(\mathscr{K})=\lim _{m}(1 / m)\left|\mathscr{K}_{m}\right|$ whenever the limit exists, where $\left|\mathscr{K}_{m}\right|$ denotes the cardinality of the set $\mathscr{K}_{m}$. A sequence $\eta=\left(\eta_{i}\right)$ is statistically convergent to $\mathscr{L}$ if for every $\varepsilon>0$

$$
\lim _{m} \frac{1}{m}\left|\left\{i \leq m:\left|\eta_{i}-\mathscr{L}\right| \geq \varepsilon\right\}\right|=0,
$$

and we write $s t-\lim _{m} \eta_{m}=\mathscr{L}$.

Let $T=\left(t_{n j}\right)$ be a matrix and $\eta=\left(\eta_{j}\right)$ be a sequence. The $T$ - transform of the sequence $\eta=\left(\eta_{n}\right)$ is defined by $T \eta=$ $\left(T_{n}(\eta)\right),(T \eta)_{n}=\sum_{j} t_{n j} \eta_{j}$ if the series converges for every $n$ $\in \mathbb{N}$. We say that $\eta$ is $T-$ summable to the number $\mathscr{L}$ if
$(T \eta)_{n}$ converges to $\mathscr{L}$. The summability matrix $T$ is regular whenever $\lim _{j} \eta_{j}=\mathscr{L}=\lim _{n}(T \eta)_{n}$.

Let $T=\left(t_{n j}\right)$ be a regular matrix. A sequence $\eta=\left(\eta_{j}\right)$ is said to be $T$-statistically convergent (see [1]) to real number $\mathscr{L}$ if for any $>0, \lim _{n} \sum_{j:\left|\eta_{j}-\mathscr{L}\right| \geq \varepsilon} t_{n j}=0$, and we write $s t_{T}-$ $\lim \eta=\mathscr{L}$. If $T$ is Cesàro matrix of order 1 , then $T$-statistical convergence is reduced to the statistical convergence.

In this paper, we also use the power series summability method which includes several known summability methods such as Abel and Borel (see [2-9]). Note that the power method is more effective than the ordinary convergence (see [10]).

Let $\left(p_{j}\right)$ be a sequence of real numbers such that $p_{0}>0$, $p_{1}, p_{2}, \cdots \geq 0$, and the corresponding power series $p(u)=$ $\sum_{j=0}^{\infty} p_{j} u^{j}$ has radius of convergence $R$ with $0<R \leq \infty$. If $\lim _{u \rightarrow R^{-}}(1 / p(u)) \sum_{j=0}^{\infty} \eta_{j} p_{j} u^{j}=L$ for all $t \in(0, R)$, then we say that $\eta=\left(\eta_{j}\right)$ is convergent in the sense of power series 
method (see $[11,12]$ ). Define $A(u) B(\eta u)=\sum_{k \geq 0} p_{k}(\eta) u^{k}$, where $A(u)=\sum_{j \geq 0} a_{j} u^{j}$ and $B(u)=\sum_{j \geq 0} b_{j} u^{j}$ are analytical functions such that $a_{0} \neq 0$ and $b_{j} \neq 0$ for all $j \geq 0$ (see [13]). Clearly, $p_{k}(\eta)=\sum_{j=0}^{k} a_{k-j} b_{j} \eta^{j}$. Moreover, the power series method is regular if and only if $\lim _{u \rightarrow R^{-}}\left(p_{j} u^{j} / p(u)\right)=0$ holds for each $j \in\{0,1, \cdots\}$ (see [14]).

We study a Korovkin-type theorem for the KantorovichStancu-type Szász-Mirakyan operators via power series method. We determine the rate of convergence for these operators. Furthermore, we give a Voronovskaya-type theorem for $T$ - statistical convergence. Such type of operators is widely studied by several authors (see [15-19]).

We start by recalling the class of KantorovichStancu-type generalization of Szász-Mirakyan operators, including Brenke-type polynomials. For every $h \in C_{B}[0, \infty)=$ $C[0, \infty) \cap E$,

$$
K_{n}^{\alpha, \beta}(h, \eta)=\frac{n+\beta}{A(1) B(n x)} \sum_{k=0}^{\infty} p_{k}(n \eta) \int_{(k+\alpha) /(n+\beta)}^{(k+1+\alpha) /(n+\beta)} h(u) d u,
$$

for $n \in \mathbb{N}, x \in 0, \infty)$ and $0 \leq \alpha \leq \beta$, where $E=\{h: x \in[0, \infty)$, $\left.\lim _{x \rightarrow \infty}\left(h(x) /\left(1+x^{2}\right)\right)<\infty\right\}$. In what follows, we calculate the moments and central moments for Kantorovich-Stancu of Szász-Mirakyan operators. Let us mention some properties of the functions $A(u)$ and $B(u)$ (see $[13,20])$.

(1) $A(1) \neq 0,\left(a_{k-r} b_{r} / A(1)\right) \geq 0$, for all $0 \leq r \leq k$ and $k=$ $0,1,2, \cdots$

(2) $B:[0, \infty) \longrightarrow(0, \infty)$

(3) Series $A(u)=\sum_{r=0}^{\infty} a_{r} u^{r}$, for $a_{0} \neq 0$, and $B(u)=\sum_{r=0}^{\infty}$ $b_{r} u^{r}$, for $b_{r} \neq 0(r \geq 0)$, are convergent for $|u|<R(R$ $>1$ ) and $A(u), B(u)$ are analytic functions

The next lemma is followed immediately from the fact that $A(u) B(\eta u)=\sum_{k \geq 0} p_{k}(\eta) u^{k}$.

Lemma 1. Let $D$ be the operator $u(d / d u)$. For all $m \geq 0$,

$$
\sum_{k \geq 0} k^{m} p_{k}(\eta) t^{k}=D^{m}(A(u) B(\eta u))
$$

For example, Lemma 1 for $m=0,1,2,3,4$ gives

$$
\begin{aligned}
\sum_{k \geq 0} p_{k}(\eta)= & A(1) B(\eta), \\
\sum_{k \geq 0} k p_{k}(\eta)= & u A^{(1)}(u) B(\eta u)+\eta u A(t) B^{(1)}(\eta u), \\
\sum_{k \geq 0} k^{2} p_{k}(\eta)= & u^{2} A^{(2)}(u) B(\eta u)+A^{(1)}(u)\left(u B(\eta u)+2 \eta u^{2} B^{(1)}(\eta u)\right) \\
& +A(u)\left(\eta u B^{(1)}(\eta u)+\eta^{2} u^{2} B^{(2)}(\eta u)\right),
\end{aligned}
$$

$$
\begin{aligned}
\sum_{k \geq 0} k^{3} p_{k}(\eta)= & u^{3} A^{(3)}(u) B(\eta u)+A^{(2)}(u)\left(3 u^{2} B(\eta u)+3 \eta u^{3} B^{(1)}(\eta u)\right) \\
& +A^{(1)}(u)\left(u B(\eta u)+6 \eta u^{2} B^{(1)}(\eta u)+3 \eta^{2} u^{3} B^{(2)}(\eta u)\right) \\
& +A(u)\left(\eta u B^{(1)}(\eta u)+3 \eta^{2} u^{2} B^{(2)}(\eta u)+\eta^{3} u^{3} B^{(3)}(\eta u)\right) \\
\sum_{k \geq 0} k^{4} p_{k}(\eta)= & u^{4} A^{(4)}(u) B(\eta u)+A^{(3)}(u)\left(6 u^{3} B(\eta u)+4 \eta u^{4} B^{(1)}(\eta u)\right) \\
& +A^{(2)}(u)\left(7 u^{2} B(\eta u)+18 \eta u^{3} B^{(1)}(\eta t)+6 \eta^{2} u^{4} B^{(2)}(\eta u)\right) \\
& +A^{(1)}(u)\left(u B(\eta u)+14 \eta u^{2} B^{(1)}(\eta u)+18 \eta^{2} u^{3} B^{(2)}(\eta u)\right. \\
& \left.+4 \eta^{3} u^{4} B^{(3)}(\eta u)\right)+A(u)\left(\eta u B^{(1)}(\eta u)+7 \eta^{2} u^{2} B^{(2)}(\eta u)\right. \\
& \left.+6 \eta^{3} u^{3} B^{(3)}(\eta u)+\eta^{4} u^{4} B^{(4)}(\eta u)\right) .
\end{aligned}
$$

Theorem 2. Let $e_{i}=e_{i}(t)=t^{i}$ for all $i \geq 0$ and let $D$ be the operator $t(d / d t)$. Then,

$$
\begin{aligned}
K_{n}^{\alpha, \beta}\left(e_{i}, x\right)= & \frac{(n+\beta)^{-i}}{(i+1) A(1) B(n x)} \sum_{j=0}^{i+1}\left(\begin{array}{c}
i+1 \\
j
\end{array}\right)\left((\alpha+1)^{i+1-j}\right. \\
& \left.-\alpha^{i+1-j}\right)\left.D^{j} A(t) B(n x t)\right|_{t=1} .
\end{aligned}
$$

Proof. By the definition of the operators, we have

$$
\begin{aligned}
K_{n}^{\alpha, \beta}\left(e_{i}, x\right)= & \frac{n+\beta}{A(1) B(n x)} \sum_{k \geq 0} p_{k}(n x) \int_{(k+\alpha) /(n+\beta)}^{(k+1+\alpha) /(n+\beta)} t^{i} d t \\
= & \frac{n+\beta}{A(1) B(n x)} \sum_{k \geq 0} p_{k}(n x) \\
& \cdot\left(\frac{(k+1+\alpha)^{i+1}}{(i+1)(n+\beta)^{i+1}}-\frac{(k+\alpha)^{i+1}}{(i+1)(n+\beta)^{i+1}}\right) \\
= & \frac{(n+\beta)^{-i}}{(i+1) A(1) B(n x)} \sum_{k \geq 0} \sum_{j=0}^{i+1}\left(\begin{array}{c}
i+1 \\
j
\end{array}\right) k^{j} p_{k}(n x) \\
& \cdot\left((\alpha+1)^{i+1-j}-\alpha^{i+1-j}\right) \\
= & \frac{(n+\beta)^{-i}}{(i+1) A(1) B(n x)} \sum_{j=0}^{i+1}\left(\begin{array}{c}
i+1 \\
j
\end{array}\right) \\
& \cdot\left((\alpha+1)^{i+1-j}-\alpha^{i+1-j}\right) \sum_{k \geq 0}^{j} k^{j} p_{k}(n x) .
\end{aligned}
$$

Thus, by Lemma 1, we complete the proof.

Lemma 3 (for instance, see [21], equation (1.27)). Let $X, L$ be two operators on the set of functions defined by $X(f(u))=$ $u f(u)$ and $L(f(u))=(d / d u) f(u)$. Then,

$$
(X L)^{m}=\sum_{j=1}^{m} S(m, j) X^{j} L^{j}
$$


Moreover,

$$
\begin{aligned}
(X L)^{m}(f(u) g(u))= & \sum_{j=1}^{m} \sum_{i=0}^{j}\left(\begin{array}{l}
j \\
i
\end{array}\right) S(m, j) u^{j} \frac{d^{i}}{d u^{i}} f(u) \\
& \cdot \frac{d^{j-i}}{d u^{j-i}} g(u),
\end{aligned}
$$

where $S(m, j)$ is the Stirling number of the second kind.

Define $a_{j}=\left.\left(d^{j} / d t^{j}\right) A(t)\right|_{t=1}$ and $b_{j}=\left.\left(d^{j} / d t^{j}\right) B(t)\right|_{t=n x}$, for all $j \geq 0$. Therefore, Theorem 2 with $D=X L$ and Lemma 3 imply the following theorem.

$K_{n}^{\alpha, \beta}\left(e_{0}, x\right)=1$,

$$
\begin{aligned}
K_{n}^{\alpha, \beta}\left(e_{1}, x\right)= & \frac{2 \alpha+1}{2(n+\beta)}+\frac{a_{1}}{a_{0}(n+\beta)}+\frac{n b_{1}}{b_{0}(n+\beta)} x, \\
K_{n}^{\alpha, \beta}\left(e_{2}, x\right)= & \frac{3 \alpha^{2}+3 \alpha+1}{3(n+\beta)^{2}}+\frac{2 a_{1}(\alpha+1)+a_{2}}{a_{0}(n+\beta)^{2}}+\frac{2 n b_{1}\left(a_{0}(\alpha+1)+a_{1}\right)}{a_{0} b_{0}(n+\beta)^{2}} x+\frac{n^{2} b_{2}}{b_{0}(n+\beta)^{2}} x^{2}, \\
K_{n}^{\alpha, \beta}\left(e_{3}, x\right)= & \frac{4 \alpha^{3}+6 \alpha^{2}+4 \alpha+1}{4(n+\beta)^{3}}+\frac{6 a_{1}(\alpha+1)^{2}+6 a_{2}(\alpha+1)+a_{1}+3 a 2+2 a_{3}}{2 a_{0}(n+\beta)^{3}}+\frac{n b_{1}\left(6 a_{0}(\alpha+1)^{2}+12 a_{1}(\alpha+1)+a_{0}+6 a_{1}+6 a_{2}\right)}{2 a_{0} b_{0}(n+\beta)^{3}} x \\
& +\frac{3 n^{2} b_{2}\left(2 a_{0}(\alpha+1)+a_{0}+2 a_{1}\right)}{2 a_{0} b_{0}(n+\beta)^{3}}+\frac{n^{3} b_{3}}{b_{0}(n+\beta)^{3}} x^{3}, \\
K_{n}^{\alpha, \beta}\left(e_{4}, x\right)= & \frac{5 \alpha^{4}+10 \alpha^{3}+10 \alpha^{2}+5 \alpha+1}{5(n+\beta)^{4}}+\frac{4 a_{1}(\alpha+1)^{3}+6 a_{2}(\alpha+1)^{2}+2\left(a_{1}+3 a_{2}+2 a_{3}\right)(\alpha+1)+3 a_{2}+4 a_{3}+a_{4}}{a_{0}(n+\beta)^{4}} \\
& +\frac{2 n b_{1}\left(2 a_{0}(\alpha+1)^{3}+6 a_{1}(\alpha+1)^{2}+\left(a_{0}+6 a_{1}+6 a_{2}\right)(\alpha+1)+3 a_{1}+6 a_{2}+2 a_{3}\right)}{a_{0} b_{0}(n+\beta)^{4}} x \\
& +\frac{3 n^{2} b_{2}\left(2 a_{0}(\alpha+1)^{2}+2\left(a_{0}+2 a_{1}\right)(\alpha+1)+a_{0}+4 a_{1}+2 a_{2}\right)}{a_{0} b_{0}(n+\beta)^{4}} x^{2}+\frac{4 n^{3} b_{3}\left(a_{0}(\alpha+1)+a_{0}+a_{1}\right)}{a_{0} b_{0}(n+\beta)^{4}} x^{3}
\end{aligned}
$$

Example 5. By applying Theorem 4 for $i=0,1,2,3,4$ with using (2), we obtain where $S(m, \ell)$ is the Stirling number of the second kind.

$$
\begin{aligned}
K_{n}^{\alpha, \beta}\left(e_{i}, x\right)= & \frac{(n+\beta)^{-i}}{i+1}\left((\alpha+1)^{i+1}-\alpha^{i+1}\right) \\
& +\frac{(n+\beta)^{-i}}{i+1} \sum_{j=1}^{i+1} \sum_{\ell=1}^{j} \sum_{s=0}^{\ell}\left(\begin{array}{c}
i+1 \\
j
\end{array}\right)\left(\begin{array}{c}
\ell \\
s
\end{array}\right) \\
& \cdot S(j, \ell)\left((\alpha+1)^{i+1-j}-\alpha^{i+1-j}\right) \frac{n^{\ell-s} a_{s} b_{\ell-s}}{a_{0} b_{0}} x^{2 \ell-s},
\end{aligned}
$$

Theorem 4. Let $e_{i}=e_{i}(t)=t^{i}$ for all $i \geq 0$. Then,

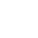

\section{.}




$$
\begin{aligned}
&= \frac{(n+\beta)^{-i}}{(i+1) A(1) B(n x)} \sum_{k \geq 0} \sum_{j=0}^{i+1}\left(\begin{array}{c}
i+1 \\
j
\end{array}\right) \\
& \cdot k^{j} p_{k}(n x)\left((1+y)^{i+1-j}-y^{i+1-j}\right) \\
&=\frac{(n+\beta)^{-i}}{(i+1) A(1) B(n x)} \sum_{j=0}^{i+1}\left(\begin{array}{c}
i+1 \\
j
\end{array}\right) \\
& \cdot\left((1+y)^{i+1-j}-y^{i+1-j}\right) \sum_{k \geq 0} k^{j} p_{k}(n x) .
\end{aligned}
$$

Thus, Lemma 1 completes the proof.

By Theorem 6 and Lemma 3, we obtain the following result.
Theorem 7. Let $y=\alpha-x(n+\beta)$. Then,

$$
\begin{aligned}
K_{n}^{\alpha, \beta}\left((t-x)^{i}, x\right)= & \frac{(n+\beta)^{-i}}{i+1}\left((y+1)^{i+1}-y^{i+1}\right) \\
& +\frac{(n+\beta)^{-i}}{i+1} \sum_{j=1}^{i+1} \sum_{\ell=1}^{j} \sum_{s=0}^{\ell}\left(\begin{array}{c}
i+1 \\
j
\end{array}\right)\left(\begin{array}{c}
\ell \\
s
\end{array}\right) \\
& \cdot S(j, \ell)\left((y+1)^{i+1-j}-y^{i+1-j}\right) \frac{n^{\ell-s} a_{s} b_{\ell-s}}{a_{0} b_{0}} x^{2 \ell-s},
\end{aligned}
$$

where $S(m, \ell)$ is the Stirling number of the second kind.

Remark 8. By applying Theorem 7 for $i=0,1,2,3$, we obtain

$$
\begin{aligned}
& K_{n}^{\alpha, \beta}(1, x)=1, \\
& K_{n}^{\alpha, \beta}(t-x, x)=\frac{2 a_{0} \alpha+a_{0}+2 a_{1}}{2 a_{0}(n+\beta)}-\frac{\left(b_{0} n+b_{0} \beta-b_{1} n\right)}{b_{0}(n+\beta)} x, \\
& K_{n}^{\alpha, \beta}\left((t-x)^{2}, x\right)=\frac{3 a_{0} \alpha^{2}+3 a_{0} \alpha+6 a_{1} \alpha+a_{0}+6 a_{1}+3 a_{2}}{3 a_{0}(n+\beta)^{2}}-\frac{2 a_{0} b_{0} \alpha(n+\beta)-2 a_{0} b_{1} n(\alpha+1)+a_{0} b_{0}(n+\beta)+2 a_{1} b_{0}(n+\beta)-2 a_{1} b_{1} n}{a_{0} b_{0}(n+\beta)^{2}} x \\
& +\frac{b_{0} n^{2}+2 b_{0} n \beta+b_{0} \beta^{2}-2 b_{1} n^{2}-2 b_{1} n \beta+b_{2} n^{2}}{b_{0}(n+\beta)^{2}} x^{2} \\
& K_{n}^{\alpha, \beta}\left((t-x)^{3}, x\right)=\frac{4 a_{0} \alpha^{3}+6 a_{0} \alpha^{2}+12 a_{1} \alpha^{2}+4 a_{0} \alpha+24 a_{1} \alpha+12 a_{2} \alpha+a_{0}+14 a_{1}+18 a_{2}+4 a_{3}}{4 a_{0}(n+\beta)^{3}} \\
& -\frac{6 a_{0} b_{0} n \alpha^{2}+6 a_{0} b_{0} \alpha^{2} \beta-6 a_{0} b_{1} n \alpha^{2}+6 a_{0} b_{0} n \alpha+6 a_{0} b_{0} \alpha \beta}{2 a_{0} b_{0}(n+\beta)^{3}} x \\
& -\frac{-12 a_{0} b_{1} n \alpha+12 a_{1} b_{0} n \alpha+12 a_{1} b_{0} \alpha \beta-12 a_{1} b_{1} n \alpha+2 a_{0} b_{0} n+2 a_{0} b_{0} \beta}{2 a_{0} b_{0}(n+\beta)^{3}} x \\
& -\frac{-7 a_{0} b_{1} n+12 a_{1} b_{0} n+12 a_{1} b_{0} \beta-18 a_{1} b_{1} n+6 a_{2} b_{0} n+6 a_{2} b_{0} \beta-6 a_{2} b_{1} n}{2 a_{0} b_{0}(n+\beta)^{3}} x \\
& +\frac{3\left(2 a_{0} b_{0} n^{2} \alpha+4 a_{0} b_{0} n \alpha \beta+2 a_{0} b_{0} \alpha \beta^{2}-4 a_{0} b_{1} n^{2} \alpha-4 a_{0} b_{1} n \alpha \beta+2 a_{0} b_{2} n^{2} \alpha\right)}{2 a_{0} b_{0}(n+\beta)^{3}} x^{2} \\
& +\frac{3\left(a_{0} b_{0} n^{2}+2 a_{0} b_{0} n \beta+a_{0} b_{0} \beta^{2}-4 a_{0} b_{1} n^{2}-4 a_{0} b_{1} n \beta+3 a_{0} b_{2} n^{2}+2 a_{1} b_{0} n^{2}\right)}{2 a_{0} b_{0}(n+\beta)^{3}} x^{2} \\
& -\frac{b_{0} n^{3}+3 b_{0} n^{2} \beta+3 b_{0} n \beta^{2}+b_{0} \beta^{3}-3 b_{1} n^{3}-6 b_{1} n^{2} \beta-3 b_{1} n \beta^{2}+3 b_{2} n^{3}+3 b_{2} n^{2} \beta-b_{3} n^{3}}{b_{0}(n+\beta)^{3}} x^{3} \text {. }
\end{aligned}
$$

Theorem 6 for $i=0,1, \cdots, 6$ (with the help of mathematical programming), we obtain the following result.

Proposition 9. Let us consider that

$$
\lim _{n \rightarrow \infty} \frac{B^{(m)}(n x)}{B(n x)}=1 \quad \text { for } \quad m=0,1,2, \cdots, 6
$$

where $B^{(m)}(t)$ is the $m$-th derivative of $B(t)$. Then, we obtain

$$
\begin{aligned}
\lim _{n \rightarrow \infty} K_{n}^{\alpha, \beta}(1, x) & =1 \\
\lim _{n \rightarrow \infty} n K_{n}^{\alpha, \beta}\left((t-x)^{1}, x\right) & =a_{1} / a_{0}+1 / 2+\alpha-\beta x \\
\lim _{n \rightarrow \infty} n K_{n}^{\alpha, \beta}\left((t-x)^{2}, x\right) & =x
\end{aligned}
$$


TABLE 1: The values of the functions $20 K_{20}^{\alpha, \beta}(f ; x)$ and $100 K_{100}^{\alpha, \beta}(f ; x)$ at $x=0,0.1,0.2, \cdots, 1$.

\begin{tabular}{lcccccrr}
\hline$x$ & 0 & 0.1 & 0.2 & 0.3 & 0.4 & 0.5 & 0.6 \\
\hline $20 K_{20}^{\alpha, \beta}(f ; x)$ & 0.01511 & 0.10173 & 0.18926 & 0.27770 & 0.36704 & 0.45729 & 0.54845 \\
$100 K_{100}^{\alpha, \beta}(f ; x)$ & 0.00326 & 0.10041 & 0.19775 & 0.29529 & 0.39303 & 0.49096 & 0.58909 \\
\hline
\end{tabular}

$$
\begin{aligned}
\lim _{n \rightarrow \infty} n^{2} K_{n}^{\alpha, \beta}\left((t-x)^{3}, x\right)= & 3 \alpha+5 / 2+3 a_{1} / a_{0}-3 \beta x^{2} \\
\lim _{n \rightarrow \infty} n^{2} K_{n}^{\alpha, \beta}\left((t-x)^{4}, x\right)= & 3 x^{2} \\
\lim _{n \rightarrow \infty} n^{3} K_{n}^{\alpha, \beta}\left((t-x)^{5}, x\right)= & \left(15 \alpha+35 / 2+15 a_{1} / a_{0}\right) x^{2} \\
& -15 \beta x^{3} \\
\lim _{n \rightarrow \infty} n^{3} K_{n}^{\alpha, \beta}\left((t-x)^{6}, x\right)= & 15 x^{3} .
\end{aligned}
$$

Example 10. Let $A(t)=1, B(t)=e^{t}, \alpha=0, \beta=1$, and $f(t)=$ $(t-x)^{2}$. By the fact that $A(t) B(x t)=\sum_{k \geq 0} p_{k}(x) t^{k}$, we have that $p_{k}(x)=x^{k} / k !$.

Table 1 presents the values of the functions $n K_{n}^{\alpha, \beta}(f ; x)$ and $x$ at $x=0,0.1,0.2, \cdots, 0.6$ and $n=20, n=100$, where we approximated $K_{n}^{\alpha, \beta}(f ; x)$ as

$$
K_{n}^{\alpha, \beta}(f, x)=\frac{n+\beta}{A(1) B(n x)} \sum_{k=0}^{3000} p_{k}(n x) \int_{(k+\alpha) /(n+\beta)}^{(k+1+\alpha) /(n+\beta)} f(t) d t .
$$

We note that the Korovkin-type theorems are very useful tools in approximation which were studied in several function spaces $[3-8,10,22-29]$. We say that sequence of operators $K_{n}^{\alpha, \beta}$ converges to $L$ in the sense of power series if

$$
\lim _{u \rightarrow R^{-}} \frac{1}{p(u)} \sum_{n=0}^{\infty} K_{n}^{\alpha, \beta}(f, x) p_{n} u^{n}=L
$$

for every $u \in(0, R)$.

\section{Main Results}

We study here $T$ - statistical convergence of the operators $K_{n}^{\alpha, \beta}$. Note that the Korovkin-type theorem for $T$ - statistical convergence was considered in [24] as follows:

Theorem 11. Let $\left(B_{j}\right)$ be a sequence of positive linear operators on $C[0,1]$ and let $T=\left(t_{n j}\right)$ be a nonnegative regular summability matrix such that

$$
s t_{T}-\lim _{n}\left\|B_{j} e_{i}-e_{i}\right\|=0, i=0,1,2 .
$$

Then, for any $f \in C[0,1]$

$$
s t_{T}-\lim _{n}\left\|B_{j} h-h\right\|=0,
$$

where $\|h\|=\max _{0 \leq x \leq 1}|h(x)|$.
Based on the above theorem, we give the following result.

Theorem 12. Let $T=\left(t_{n j}\right)$ be a regular matrix and $\left(K_{n}^{\alpha, \beta}\right)$ be as in (2) on $C[0, M] \cap E$ such that $\lim _{n \rightarrow \infty}\left(B^{(i)}(n x)\right)$ / $(B(n x))=1$, where $B^{(i)}(n x)$ denotes $i^{\text {th }}$ derivative and

$$
s t_{A}-\lim _{n}\left\|K_{n}^{\alpha, \beta} e_{i}-e_{i}\right\|=0(i=1,2) .
$$

Then, for any $h \in C[0, M] \cap E$

$$
s t_{A}-\lim _{n}\left\|K_{n}^{\alpha, \beta} h-h\right\|=0,
$$

where $\|h\|=\max _{t \in[0, M]}|h(t)|$.

Proof. From Lemma 5, we have that $s_{T}-\lim _{n}\left\|K_{n}^{\alpha, \beta} e_{0}-e_{0}\right\|$ $=0$. Now, we will estimate the following expressions:

$$
\begin{aligned}
\left\|K_{n}^{\alpha, \beta} e_{1}-e_{1}\right\| \leq & \left\|x\left(\frac{n}{n+\beta} \cdot \frac{B^{\prime}(n x)}{B(n x)}-1\right)\right\| \\
& +\left\|\frac{A^{\prime}(1)}{(n+\beta) A(1)}\right\|+\left\|\frac{2 \alpha+1}{2(n+\beta)}\right\| .
\end{aligned}
$$

Note that $\lim _{n \rightarrow \infty}\left(B^{\prime}(n x)\right) /(B(n x))=1$. So from the last two relations we have that $\left\|K_{n}^{\alpha, \beta} e_{1}-e_{1}\right\|=0$. Moreover,

$$
\begin{aligned}
\left\|K_{n}^{\alpha, \beta} e_{2}-e_{2}\right\|= & \|\left(\frac{n}{n+\beta}\right)^{2} \frac{B^{\prime \prime}(n x)}{B(n x)} \cdot x^{2} \\
& +\frac{n B^{\prime}(n x)\left[2 A^{\prime}(1)+(2 \alpha+2) A(1)\right]}{(n+\beta)^{2} A(1) B(n x)} x \\
& +\frac{1}{(n+\beta)^{2} A(1)}\left\{A^{\prime \prime}(1)+(2 \alpha+2) A^{\prime}(1)\right. \\
& \left.\cdot\left(\alpha^{2}+\alpha+\frac{1}{3}\right) A(1)\right\}-x^{2} \| \longrightarrow 0 .
\end{aligned}
$$

Now proof follows directly from Theorem 11 .

This theorem is an extension of some known results for the Kantorovich-Stancu-type Szász-Mirakyan operators. 
Example 13 (see [6]). Under conditions given in Theorem 12, we define the following operators

$$
N_{n}(h, x)=\left(1+x_{n}\right) K_{n}^{\alpha, \beta}(h, x),
$$

where the sequence $\left(x_{n}\right)$ is given as follows:

$$
\left(x_{n}\right)= \begin{cases}\frac{1}{n^{3}} ; & m^{2}-m \leq n \leq m^{2}-1 \\ \frac{1}{n^{4}} ; & n=m^{2} ; m \in \mathbb{N} \backslash\{1\} \\ 0 ; & \text { otherwise, }\end{cases}
$$

then

$$
\begin{aligned}
N_{n}\left(e_{0}, x\right)= & \left(1+x_{n}\right), \\
N_{n}\left(e_{1}, x\right)= & \left(1+x_{n}\right)\left(\frac{2 \alpha+1}{2(n+\beta)}+\frac{a_{1}}{a_{0}(n+\beta)}+\frac{n b_{1}}{b_{0}(n+\beta)} x\right), \\
N_{n}\left(e_{2}, x\right)= & \left(1+x_{n}\right)\left(\frac{3 \alpha^{2}+3 \alpha+1}{3(n+\beta)^{2}}+\frac{2 a_{1}(\alpha+1)+a_{2}}{a_{0}(n+\beta)^{2}}\right. \\
& \left.+\frac{2 n b_{1}\left(a_{0}(\alpha+1)+a_{1}\right)}{a_{0} b_{0}(n+\beta)^{2}} x+\frac{n^{2} b_{2}}{b_{0}(n+\beta)^{2}} x^{2}\right) .
\end{aligned}
$$

By Theorem 11 we obtain $s t_{T}-\lim _{n}\left\|N_{n} h-h\right\|=0$, but the operators $N_{n}(h, x)$ do not satisfy Theorem 12 . Hence, the sequence $\left(N_{n}\right)$ is not statistically convergent but it is $T$ - statistically convergent.

Remark 14 . The sequence $\left(x_{n}\right)$ is not statistically convergent and hence not convergent. As an example, consider the Cesáro matrix of order 2 .

$$
T=\left(t_{n k}\right)= \begin{cases}\frac{2(n+1-k)}{(n+1)(n+2)} ; & 0 \leq k \leq n, \\ 0 ; & k>n,\end{cases}
$$

where

$$
\begin{aligned}
0 \leq \lim _{n} \sum_{k:\left|x_{k}-\alpha\right| \geq \varepsilon} t_{n k} & =\lim _{n} \sum_{\substack{k=m^{2}-m, \cdots, m^{2}-1 \\
k=m^{2} ; m \in \mathbb{N} \backslash\{1\}}} t_{n k} \\
& =\lim _{n} \frac{2}{(n+1)(n+2)}[1+\cdots+n] \\
& \leq \lim _{n} \frac{2}{(n+1)(n+2)} \cdot \frac{n(n+1)}{2}=1 .
\end{aligned}
$$

This proves that $x=\left(x_{n}\right)$ is $T$ - statistically convergent. We have

$$
\begin{aligned}
N_{n}\left(e_{0}, x\right)= & \left(1+x_{n}\right), \\
N_{n}\left(e_{1}, x\right)= & \left(1+x_{n}\right)\left(\frac{2 \alpha+1}{2(n+\beta)}+\frac{a_{1}}{a_{0}(n+\beta)}+\frac{n b_{1}}{b_{0}(n+\beta)} x\right), \\
N_{n}\left(e_{2}, x\right)= & \left(1+x_{n}\right)\left(\frac{3 \alpha^{2}+3 \alpha+1}{3(n+\beta)^{2}}+\frac{2 a_{1}(\alpha+1)+a_{2}}{a_{0}(n+\beta)^{2}}\right. \\
& \left.+\frac{2 n b_{1}\left(a_{0}(\alpha+1)+a_{1}\right)}{a_{0} b_{0}(n+\beta)^{2}} x+\frac{n^{2} b_{2}}{b_{0}(n+\beta)^{2}} x^{2}\right) .
\end{aligned}
$$

By Example 13, this shows that $N_{n}(h, x)$ does not satisfy Theorem 12.

In $[27,29]$, Korovkin-type theorems are proved by Abel summability method. Now, we discuss for power series method. Let $B[0, \infty)(C[0, \infty))$ be the space of all bounded (continuous) functions on the interval $[0, \infty)$.

Theorem 15. Let $\left(K_{n}^{\alpha, \beta}\right)$ be a sequence of positive linear operators from $C[0, M] \cap E$ into $B[0, M] \cap E$ such that

$$
\lim _{t \rightarrow R^{-}} \frac{1}{p(t)}\left\|\sum_{n=0}^{\infty}\left(K_{n}^{\alpha, \beta} e_{i}-e_{i}\right) p_{n} t^{n}\right\|=0, i=0,1,2 .
$$

Then, for $\mathfrak{h} \in C[0, M] \cap E$,

$$
\lim _{t \rightarrow R^{-}} \frac{1}{p(t)}\left\|\sum_{n=0}^{\infty}\left(K_{n}^{\alpha, \beta} \mathfrak{h}-\mathfrak{h}\right) p_{n} t^{n}\right\|=0 .
$$

Proof. Clearly, from (32) follows (31). Now, we show the converse that (31) implies (32). Let $\mathfrak{h} \in C[0, M] \cap E$, then there exists a constant $K>0$ such that $|\mathfrak{h}(u)| \leq K$ for all $u \in 0, M]$. Therefore,

$$
|\mathfrak{h}(u)-\mathfrak{h}(x)|[\in 0, M]
$$

For every given $\varepsilon>0$, there exists $\delta>0$ such that

$$
|\mathfrak{h}(u)-\mathfrak{h}(x)| \leq \varepsilon,
$$

whenever $|u-x|<\delta$ for all $u \in 0, M]$. Define $\psi \equiv \psi(u, x)=$ $(u-x)^{2}$. If $|u-x| \geq \delta$, then

$$
|\mathfrak{h}(u)-\mathfrak{h}(x)| \leq \frac{2 K}{\delta^{2}} \psi(u, x) .
$$

From (33)-(35), we have that $|\mathfrak{h}(u)-\mathfrak{h}(x)|<\varepsilon+(2 K /$ $\left.\delta^{2}\right) \psi(u, x)$, namely,

$$
-\varepsilon-\frac{2 K}{\delta^{2}} \psi(u, x)<\mathfrak{h}(t)-\mathfrak{h}(x)<\frac{2 K}{\delta^{2}} \psi(u, x)+\varepsilon .
$$


By applying the operator $K_{n}^{\alpha, \beta}(1, x), K_{n}^{\alpha, \beta}(1, x)$ is a monotone and linear operator, we obtain

$$
\begin{aligned}
K_{n}^{\alpha, \beta}(1, x)\left(-\varepsilon-\frac{2 K}{\delta^{2}} \psi\right) & <K_{n}^{\alpha, \beta}(1, x)(\mathfrak{h}(u)-\mathfrak{h}(x)) \\
& <K_{n}^{\alpha, \beta}(1, x)\left(\frac{2 K}{\delta^{2}} \psi+\varepsilon\right)
\end{aligned}
$$

which implies

$$
\begin{aligned}
-\varepsilon & K_{n}^{\alpha, \beta}(1, x)-\frac{2 K}{\delta^{2}} K_{n}^{\alpha, \beta}(\psi(u), x) \\
< & K_{n}^{\alpha, \beta}(\mathfrak{h}, x)-\mathfrak{h}(x) K_{n}^{\alpha, \beta}(1, x) \\
& <\frac{2 K}{\delta^{2}} K_{n}^{\alpha, \beta}(\psi(u), x)+\varepsilon K_{n}^{\alpha, \beta}(1, x) .
\end{aligned}
$$

On the other hand,

$$
\begin{aligned}
K_{n}^{\alpha, \beta}(\mathfrak{h}, x)-\mathfrak{h}(x)= & K_{n}^{\alpha, \beta}(\mathfrak{h}, x)-\mathfrak{h}(x) K_{n}^{\alpha, \beta}(1, x) \\
& +\mathfrak{h}(x)\left[K_{n}^{\alpha, \beta}(1, x)-1\right] .
\end{aligned}
$$

From (38) and (39) we get

$$
\begin{aligned}
K_{n}^{\alpha, \beta}(\mathfrak{h}, x)-\mathfrak{h}(x)< & \frac{2 K}{\delta^{2}} K_{n}^{\alpha, \beta}(\psi(u), x)+\varepsilon K_{n}^{\alpha, \beta}(1, x) \\
& +\mathfrak{h}(x)\left[K_{n}^{\alpha, \beta}(1, x)-1\right] .
\end{aligned}
$$

Now, we estimate the following expression:

$$
\begin{aligned}
K_{n}^{\alpha, \beta}(\psi(u), x) & =K_{n}^{\alpha, \beta}\left((x-u)^{2}, x\right)=K_{n}^{\alpha, \beta}\left(\left(x^{2}-2 x u+u^{2}\right), x\right) \\
& =x^{2} K_{n}^{\alpha, \beta}(1, x)-2 x K_{n}^{\alpha, \beta}(u, x)+K_{n}^{\alpha, \beta}\left(u^{2}, x\right) .
\end{aligned}
$$

By (40), we obtain

$$
\begin{aligned}
& K_{n}^{\alpha, \beta}(\mathfrak{h}, x)-\mathfrak{h}(x) \\
&< \frac{2 K}{\delta^{2}}\left\{x^{2}\left[K_{n}^{\alpha, \beta}(1, x)-1\right]-2 x\left[K_{n}^{\alpha, \beta}(u, x)-x\right]\right. \\
&\left.+\left[K_{n}^{\alpha, \beta}\left(u^{2}, x\right)-x^{2}\right]\right\}+\varepsilon K_{n}^{\alpha, \beta}(1, x) \\
&+f(x)\left[K_{n}^{\alpha, \beta}(1, x)-1\right] \\
&= \varepsilon+\varepsilon\left[K_{n}^{\alpha, \beta}(1, x)-1\right]+\mathfrak{h}(x)\left[K_{n}^{\alpha, \beta}(1, x)-1\right]++\frac{2 K}{\delta^{2}} \\
& \cdot\left\{x^{2}\left[K_{n}^{\alpha, \beta}(1, x)-1\right]-2 x\left[K_{n}^{\alpha, \beta}(u, x)-x\right]\right. \\
&\left.+\left[K_{n}^{\alpha, \beta}\left(u^{2}, x\right)-x^{2}\right]\right\} .
\end{aligned}
$$

Therefore,

$$
\begin{aligned}
\left|K_{n}^{\alpha, \beta}(\mathfrak{h}, x)-\mathfrak{h}(x)\right| \leq & +\left(\varepsilon+K+\frac{2 K M^{2}}{\delta^{2}}\right)\left|K_{n}^{\alpha, \beta}(1, x)-1\right| \\
& +\frac{4 K M}{\delta^{2}}\left|K_{n}^{\alpha, \beta}(u, x)-x\right| \\
& +\frac{2 K}{\delta^{2}}\left|K_{n}^{\alpha, \beta}\left(u^{2}, x\right)-x^{2}\right| .
\end{aligned}
$$

From the above relations and the linearity of $K_{n}^{\alpha, \beta}$, we obtain

$$
\begin{aligned}
\frac{1}{p(v)} \| & \sum_{n=0}^{\infty}\left(U_{n, p}(\mathfrak{h} ; x)-\mathfrak{h}(x)\right) p_{n} v^{n} \| \\
\leq & \left.\varepsilon+\left(\varepsilon+K+\frac{2 K M^{2}}{\delta^{2}}\right) \frac{1}{p(t)} \| \sum_{n=0}^{\infty}\left(K_{n}^{\alpha, \beta}(1 ; x)-1\right)\right) p_{n} t^{n} \| \\
& \left.+\frac{4 K M}{\delta^{2}} \frac{1}{p(v)} \| \sum_{n=0}^{\infty}\left(K_{n}^{\alpha, \beta}(u ; x)-x\right)\right) p_{n} v^{n} \| \\
& +\frac{2 K}{\delta^{2}} \frac{1}{p(v)}\left\|\sum_{n=0}^{\infty}\left(K_{n}^{\alpha, \beta}\left(u^{2} ; x\right)-x^{2}\right) p_{n} v^{n}\right\| .
\end{aligned}
$$

Hence, (32) follows from the last relation and (31).

\section{Rate of Convergence}

Modulus of continuity is defined by

$$
\omega(\mathfrak{h}, \delta)=\sup _{|h|<\delta}|\mathfrak{h}(x+h)-\mathfrak{h}(x)|, \mathfrak{h}(x) \in C[0, M] \cap E .
$$

It is not hard to verify

$$
|\mathfrak{h}(x)-\mathfrak{h}(y)| \leq \omega(\mathfrak{h}, \delta)\left(\frac{|x-y|}{\delta}+1\right)
$$

So, we can state the following.

Theorem 16. Let $T=\left(t_{i j}\right)$ be a nonnegative regular summability matrix and $\mathfrak{h} \in C[0, M] \cap E$. If $\left(\alpha_{n}\right)$ is a sequence of positive real numbers such that $\omega\left(\mathfrak{h}, \delta_{n}\right)=s t_{T}-O\left(\alpha_{n}\right)$, then

$$
\left\|K_{n}^{\alpha, \beta} \mathfrak{h}-\mathfrak{h}\right\|=s t_{A}-O\left(\alpha_{n}\right),
$$


where

$$
\begin{aligned}
\delta_{n}= & \left\{M^{2}\left\|\left(\frac{n^{2} B^{\prime \prime}(n x)}{(n+\beta)^{2} B(n x)}-\frac{2 n B^{\prime}(n x)}{(n+\beta) B(n x)}+1\right)\right\|\right. \\
& +M \|\left(\frac{n B^{\prime}(n x)\left[2^{\prime}(1)+(2 \alpha+2) A(1)\right]}{(n+\beta)^{2} A(1) B(n x)}\right. \\
& \left.-\frac{2 A^{\prime}(1)}{(n+\beta) A(1)}+\frac{2(2 \alpha+1)}{2(n+\beta)}\right)\|+\| \frac{1}{(n+\beta)^{2} A(1)} \\
& \left.\cdot\left\{A^{\prime^{\prime}}(1)+(2 \alpha+2) A^{\prime}(1)\left(\alpha^{2}+\alpha+\frac{1}{3}\right) A(1)\right\} \|\right\}^{2},
\end{aligned}
$$

for any positive integer $n$.

Proof. Let $\mathfrak{h} \in C[0, M] \cap E$. By positivity and linearity of $K_{n}^{\alpha, \beta}$ and (46), we see

$$
\begin{aligned}
\left|K_{n}^{\alpha, \beta}(\mathfrak{h} ; x)-\mathfrak{h}\right| \leq & K_{n}^{\alpha, \beta}(|\mathfrak{h}(t)-\mathfrak{h}(x)| ; x) \\
\leq & \frac{n+\beta}{A(1) B(n x)} \sum_{k=0}^{\infty} p_{k}(n x) \int_{(k+\alpha) /(n+\beta)}^{(k+1+\alpha) /(n+\beta)} \omega(\mathfrak{h}, \delta) \\
& \cdot\left(1+\frac{|t-x|}{\delta}\right) d t \\
\leq & \omega(\mathfrak{h}, \delta)\left[1+\frac{1}{\delta} \frac{n+\beta}{A(1) B(n x)} \sum_{k=0}^{\infty} p_{k}(n x)\right. \\
& \left.\cdot \int_{(k+\alpha) /(n+\beta)}^{(k+1+\alpha) /(n+\beta)}(t-x) d t\right](\text { by Lemma } 1)=\omega(\mathfrak{h}, \delta) \\
& \cdot\left[1+\frac{1}{\delta} K_{n}^{\alpha, \beta}(|t-x| ; x)\right] .
\end{aligned}
$$

By applying the Cauchy-Schwartz inequality, we have

$$
\left|K_{n}^{\alpha, \beta}(\mathfrak{h} ; x)-\mathfrak{h}\right| \leq \omega(\mathfrak{h}, \delta)\left[1+\frac{1}{\delta}\left(K_{n}^{\alpha, \beta}\left(|t-x|^{2} ; x\right)\right)^{1 / 2}\right]
$$

Based on Examples 5 and Remark 8, we obtain

$$
\begin{aligned}
& K_{n}^{\alpha, \beta}\left((u-x)^{2} ; x\right) \\
& =K_{n}^{\alpha, \beta}\left(e_{2} ; x\right)-2 x K_{n}^{\alpha, \beta}\left(e_{1} ; x\right)+x^{2} K_{n}^{\alpha, \beta}\left(e_{0} ; x\right) \\
& \leq M^{2}\left\|\left(\frac{n^{2} B^{\prime \prime}(n x)}{(n+\beta)^{2} B(n x)}-\frac{2 n B^{\prime}(n x)}{(n+\beta) B(n x)}+1\right)\right\| \\
& +M \|\left(\frac{n B^{\prime}(n x)\left[2 A^{\prime}(1)+(2 \alpha+2) A(1)\right]}{(n+\beta)^{2} A(1) B(n x)}\right.
\end{aligned}
$$

$$
\begin{aligned}
& \left.-\frac{2 A^{\prime}(1)}{(n+\beta) A(1)}+\frac{2(2 \alpha+1)}{2(n+\beta)}\right)\|+\| \frac{1}{(n+\beta)^{2} A(1)} \\
& \cdot\left\{A^{\prime \prime}(1)+(2 \alpha+2) A^{\prime}(1)\left(\alpha^{2}+\alpha+\frac{1}{3}\right) A(1)\right\} \|
\end{aligned}
$$

By taking

$$
\begin{aligned}
\delta_{n}= & \left\{M^{2}\left\|\left(\frac{n^{2} B^{\prime \prime}(n x)}{(n+\beta)^{2} B(n x)}-\frac{2 n B^{\prime}(n x)}{(n+\beta) B(n x)}+1\right)\right\|\right. \\
& +M \|\left(\frac{n B^{\prime}(n x)\left[2 A^{\prime}(1)+(2 \alpha+2) A(1)\right]}{(n+\beta)^{2} A(1) B(n x)}\right. \\
& \left.-\frac{2 A^{\prime}(1)}{(n+\beta) A(1)}+\frac{2(2 \alpha+1)}{2(n+\beta)}\right)\|+\| \frac{1}{(n+\beta)^{2} A(1)} \\
& \left.\cdot\left\{A^{\prime \prime}(1)+(2 \alpha+2) A^{\prime}(1)\left(\alpha^{2}+\alpha+\frac{1}{3}\right) A(1)\right\} \|\right\}^{2},
\end{aligned}
$$

we get that $\left\|K_{n}^{\alpha, \beta} \mathfrak{h}-\mathfrak{h}\right\| \leq 2 \cdot \omega\left(\mathfrak{h}, \delta_{n}\right)$. Therefore, for every $\varepsilon>0$, we have

$$
\frac{1}{\alpha_{n}} \sum_{\left\|K_{n}^{\alpha, \beta} \mathfrak{h}-\mathfrak{h}\right\| \geq \varepsilon} t_{n j} \leq \frac{1}{\alpha_{n}} \sum_{2 \cdot \omega\left(f, \delta_{n}\right) \geq \varepsilon} t_{n j}
$$

From the conditions that are given in the theorem, we have that $\left\|K_{n}^{\alpha, \beta} \mathfrak{h}-\mathfrak{h}\right\|=s t_{T}-0\left(\alpha_{i}\right)$, as claimed.

In the next result, we present the rate of convergence for the power summability method.

Theorem 17. Let $\mathfrak{h} \in C[0, M] \cap E$ and let $\phi$ be a positive real function defined on $(0, M) \cap E$. If $\omega(\mathfrak{h}, \psi(u))=O(\phi(u))$, as $v \longrightarrow R^{-}$, then we have

$$
\frac{1}{p(v)}\left\|\sum_{n=0}^{\infty}\left(K_{n}^{\alpha, \beta} e_{i}-e_{i}\right) p_{n} v^{n}\right\|=O(\phi(v))
$$

where the function $\psi:(0, M) \cap E \longrightarrow \mathbb{R}$ is defined by relation

$$
\psi(u)=\left\{\sup _{\substack{x \in(0, M) \\ n \in \mathbb{N}}}\left\{K_{n}^{\alpha, \beta}\left((u-x)^{2} ; x\right)\right\}\right\}^{1 / 2} .
$$


Proof. Let $\mathfrak{h} \in C[0, M] \cap E$. For any $u \in(0, R), x \in(0, M)$, and $\delta>0$, we have

$$
\begin{aligned}
\left|\sum_{n=0}^{\infty}\left[K_{n}^{\alpha, \beta}(\mathfrak{h} ; x)-\mathfrak{h}(x)\right] p_{n} v^{n}\right| & \\
\leq & \sum_{n=0}^{\infty} K_{n}^{\alpha, \beta}(|\mathfrak{h}(u)-\mathfrak{h}(x)| ; x) p_{n} v^{n} \\
\leq & \sum_{n=0}^{\infty} K_{n}^{\alpha, \beta}\left(\omega\left(\mathfrak{h}, \frac{|u-x|}{\delta} \delta\right) ; x\right) p_{n} v^{n} \\
\leq & \sum_{n=0}^{\infty} K_{n}^{\alpha, \beta}\left(\left(1+\left[\left|\frac{u-x}{\delta}\right|\right]\right) \omega(\mathfrak{h}, \delta) ; x\right) p_{n} v^{n} \\
\leq & \omega(\mathfrak{h}, \delta) \sum_{n=0}^{\infty} K_{n}^{\alpha, \beta}\left(1+\frac{(u-x)^{2}}{\delta^{2}} ; x\right) p_{n} v^{n} \\
\leq & \omega(\mathfrak{h}, \delta) \sum_{n=0}^{\infty} K_{n}^{\alpha, \beta}\left(e_{0}(u) ; x\right) p_{n} v^{n} \\
& +\frac{\omega(\mathfrak{h}, \delta)}{\delta^{2}} \sum_{n=0}^{\infty} K_{n}^{\alpha, \beta}\left((u-x)^{2} ; x\right) p_{n} v^{n} \\
= & p(v) \omega(\mathfrak{h}, \delta)+\frac{\omega(\mathfrak{h}, \delta)}{\delta^{2}} \quad \begin{array}{c}
0 \leq x \leq 1 \\
\sup
\end{array} \\
& \cdot\left\{K_{n}^{\alpha, \beta}\left((u-x)^{2} ; x\right)\right\} \sum_{n=0}^{\infty} p_{n} v^{n},
\end{aligned}
$$

which leads to

$$
\begin{aligned}
\mid \sum_{n=0}^{\infty}[ & \left.K_{n}^{\alpha, \beta}(f ; x)-f(x)\right] p_{n} v^{n} \mid \\
\leq & p(v) \omega(f, \delta)+\frac{\omega(f, \delta)}{\delta^{2}} \sup _{0 \leq x \leq 1} \\
\cdot & \left\{K_{n}^{\alpha, \beta}\left((u-x)^{2} ; x\right)\right\} p(v) .
\end{aligned}
$$

If we set $\delta=\psi(u)$, then from the last inequality we have

$$
0 \leq \frac{1}{p(v)}\left\|\sum_{n=0}^{\infty}\left(K_{n}^{\alpha, \beta} \mathfrak{h}-\mathfrak{h}\right) p_{n} v^{n}\right\| \leq 2 \omega(\mathfrak{h}, \delta),
$$

as required.

\section{Voronovskaya-Type Theorems}

First, we prove a Voronovskaya-type theorem for the operators under consideration.
Theorem 18. Let $\mathfrak{h}, \mathfrak{h}^{\prime}, \mathfrak{h}^{\prime \prime} \in C[0, M] \cap E$ and $\lim _{n \rightarrow \infty} B^{(i)}$ $(n x) / B(n x)=1$, for $i \in\{1,2\}$. Then,

$$
\begin{aligned}
\lim _{n \rightarrow \infty}( & n+\beta)\left[K_{n}^{\alpha, \beta}(\mathfrak{h} ; x)-\mathfrak{h}(x)\right] \\
= & \left(\frac{A^{\prime}(1)}{A(1)}+\frac{1}{2}+\alpha-\beta x\right) \mathfrak{h}^{\prime}(x)+\frac{x}{2} \mathfrak{h}^{\prime \prime}(x),
\end{aligned}
$$

for every $x \in[0, M]$.

Proof. Assume that $\mathfrak{h}^{\prime}, \mathfrak{h}^{\prime \prime} \in C[0, M] \cap E$ and $x \in[0, M]$. By Taylor's formula, we have

$\mathfrak{h}(y)=\mathfrak{h}(x)+(y-x) \mathfrak{h}^{\prime}(x)+\frac{1}{2}(y-x)^{2} \mathfrak{h}^{\prime \prime}(x)+(y-x)^{2} \psi(y-x)$,

where $\psi(y-x) \longrightarrow 0$ and $y-x \longrightarrow 0$. Applying in both sides of the above relation operators $K_{n}^{\alpha, \beta}$, we obtain

$$
\begin{aligned}
K_{n}^{\alpha, \beta}(\mathfrak{h}, x)-\mathfrak{h}(x)= & \mathfrak{h}^{\prime}(x) K_{n}^{\alpha, \beta}(y-x ; x) \\
& +\frac{\mathfrak{h}^{\prime \prime}(x)}{2} K_{n}^{\alpha, \beta}\left((y-x)^{2} ; x\right) \\
& +K_{n}^{\alpha, \beta}\left((y-x)^{2} \psi(y-x) ; x\right),
\end{aligned}
$$

which implies

$$
\begin{aligned}
(n+\beta)\left[K_{n}^{\alpha, \beta}(\mathfrak{h}, x)-\mathfrak{h}(x)\right]= & (n+\beta) \mathfrak{h}^{\prime}(x) K_{n}^{\alpha, \beta}(y-x ; x) \\
& +(n+\beta) \frac{\mathfrak{h}^{\prime \prime}(x)}{2} K_{n}^{\alpha, \beta}\left((y-x)^{2} ; x\right) \\
& +(n+\beta) K_{n}^{\alpha, \beta}\left((y-x)^{2} \psi(y-x) ; x\right) .
\end{aligned}
$$

Now, we will estimate this expression:

$$
\lim _{n \rightarrow \infty}(n+\beta) K_{n}^{\alpha, \beta}\left((y-x)^{2} \psi(y-x) ; x\right) .
$$

Let $\varepsilon>0$ and $\delta>0$ such that $|\psi(y-x)|<\varepsilon$, where $|y-x|$ $<\delta$. We will split the above relation in two parts:

$$
\begin{aligned}
& U_{1}=(n+\beta) \frac{n+\beta}{A(1) B(n x)} \sum_{k=0}^{\infty} p_{k}(n x) \int_{|y-x| \leq \delta}(y-x)^{2} \psi(y-x) d y \\
& U_{2}=(n+\beta) \frac{n+\beta}{A(1) B(n x)} \sum_{k=0}^{\infty} p_{k}(n x) \int_{|y-x| \geq \delta}(y-x)^{2} \psi(y-x) d y .
\end{aligned}
$$




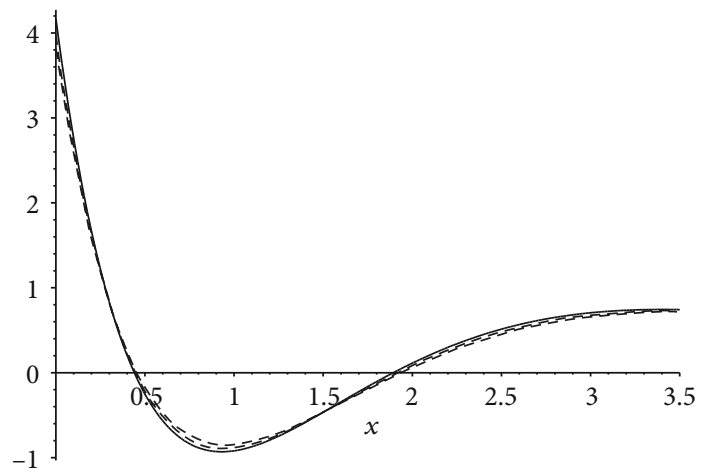

FigURE 1: The graphs of $(10+\beta)\left[K_{10}^{\alpha, \beta}(f ; x)-f(x)\right],(20+\beta)\left[K_{20}^{\alpha, \beta}(f ; x)-f(x)\right]$, and $\left(\left(A^{\prime}(1) / A(1)\right)+(1 / 2)+\alpha-\beta x\right) f^{\prime}(x)+(x / 2) f^{\prime \prime}(x)$ when $x \in 0,3.5]$.

From the above conditions, we have

$$
\begin{aligned}
\left|U_{1}\right| & \leq \varepsilon(n+\beta)^{2} \frac{1}{A(1) B(n x)} \sum_{k=0}^{\infty} p_{k}(n x) \int_{k+\alpha / n+\beta}^{k+\alpha+1 / n+\beta}(y-x)^{2} d y \\
& =\varepsilon(n+\beta) K_{n}^{\alpha, \beta}\left((y-x)^{2} ; x\right) .
\end{aligned}
$$

On the other hand, from Proposition 9, condition (3), we get that $\left|U_{1}\right| \leq \varepsilon_{1}$.

Let us denote by $N=\sup _{x}\{|\psi(y-x)| ;|y-x| \geq \delta\}$. Then, we obtain

$$
\begin{aligned}
\left|U_{2}\right| & \leq \frac{N}{\delta^{2}}(n+\beta) \frac{n+\beta}{A(1) B(n x)} \sum_{k=0}^{\infty} p_{k}(n x) \int_{|y-x| \geq \delta}(y-x)^{4} d y \\
& =\frac{N}{\delta^{2}}(n+\beta) K_{n}^{\alpha, \beta}\left((y-x)^{4} ; x\right) .
\end{aligned}
$$

Condition (5) in Proposition 9 tells us that $\lim _{n \rightarrow \infty}$ $\left|U_{2}\right|=0$, which completes the proof.
Example 19. Let $A(t)=1, B(t)=e^{t}, \alpha=1 / 3, \beta=1 / 2$, and $\mathfrak{h}(x)=5 x e^{-x}$. By $A(t) B(x t)=\sum_{k \geq 0} p_{k}(x) t^{k}$, we see that $p_{k}$ $(x)=x^{k} / k$ !. Figure 1 presents the graphs of the functions $(10+\beta)\left[K_{10}^{\alpha, \beta}(\mathfrak{h} ; x)-\mathfrak{h}(x)\right], \quad(20+\beta)\left[K_{20}^{\alpha, \beta}(\mathfrak{h} ; x)-\mathfrak{h}(x)\right]$, and $\left(\left(A^{\prime}(1) / A(1)\right)+(1 / 2)+\alpha-\beta x\right) \mathfrak{h}^{\prime}(x)+(x / 2) \mathfrak{h}^{\prime \prime}(x)$.

We extend the Voronovskaya-type theorem for $T$ - statistical method for these operators. Consider operators $N_{n}$ from Example 13. We start with the following lemma.

Lemma 20. Let $\mathfrak{h} \in C[0, M] \cap E$ such that $\mathfrak{h}^{\prime}, \mathfrak{h}^{\prime \prime} \in C[0, M] \cap$ $E, x \in[0, M]$, and $\lim _{n \rightarrow \infty}\left(B^{(i)}(n x) / B(n x)\right)=1$, for $i \in\{1,2$, $3,4\}$. Then, we obtain

$$
(n+\beta)^{4} K_{n}\left((y-x)^{4} ; x\right) \sim 0\left(s t_{A}\right) \quad \text { on } \quad[0, M] .
$$

Proof. The proposition follows directly from Proposition 9 (5).

Theorem 21. Let $\mathfrak{h} \in C[0, M] \cap E$ such that $\mathfrak{h}^{\prime}, \mathfrak{h}^{\prime \prime} \in C[0, M]$ $\cap E, x \in[0, M]$, for any finite $M$ and let $\lim _{n \rightarrow \infty}\left(B^{(i)}(n x)\right.$ $/ B(n x))=1$, for $i \in\{1,2,3,4\}$. Then, for $x \in[0, M]$,

$$
\begin{aligned}
\mid(n+ & \beta)^{2}\left[N_{n}(\mathfrak{h} ; x)-\mathfrak{h}(x)-\mathfrak{h}^{\prime}(x)\left[\frac{2 a_{0} \alpha+a_{0}+2 a_{1}}{2 a_{0}(n+\beta)}-\frac{\left(b_{0} n+b_{0} \beta-b_{1} n\right)}{b_{0}(n+\beta)} x\right]-\frac{\mathfrak{h}^{\prime \prime}(x)}{2}\right. \\
& \cdot\left(-\frac{2 a_{0} b_{0} \alpha(n+\beta)-2 a_{0} b_{1} n(\alpha+1)+a_{0} b_{0}(n+\beta)+2 a_{1} b_{0}(n+\beta)-2 a_{1} b_{1} n}{a_{0} b_{0}(n+\beta)^{2}} x\right. \\
& \left.\left.+\frac{b_{0} n^{2}+2 b_{0} n \beta+b_{0} \beta^{2}-2 b_{1} n^{2}-2 b_{1} n \beta+b_{2} n^{2}}{b_{0}(n+\beta)^{2}} x^{2}\right)\right] \mid \\
& \sim \frac{\mathfrak{h}^{\prime \prime}(x)}{2} \cdot \frac{3 a_{0} \alpha^{2}+3 a_{0} \alpha+6 a_{1} \alpha+a_{0}+6 a_{1}+3 a_{2}}{3 a_{0}(n+\beta)^{2}} .
\end{aligned}
$$


Proof. Taylor's formula gives

$\mathfrak{h}(y)=\mathfrak{h}(x)+(y-x) \mathfrak{h}^{\prime}(x)+\frac{1}{2}(y-x)^{2} \mathfrak{h}^{\prime \prime}(x)+(y-x)^{2} \psi(y-x)$, where $\psi(y-x) \longrightarrow 0$, as $y-x \longrightarrow 0$. Taking into consideration Remark 8, after applying $N_{n}$ in both sides of relation (69), we obtain

$$
\begin{aligned}
N_{n}(\mathfrak{h})= & \left(1+x_{n}\right) \mathfrak{h}(x)+\left(1+x_{n}\right) \mathfrak{h}^{\prime}(x)\left(\frac{2 a_{0} \alpha+a_{0}+2 a_{1}}{2 a_{0}(n+\beta)}-\frac{\left(b_{0} n+b_{0} \beta-b_{1} n\right)}{b_{0}(n+\beta)} x\right)+\left(1+x_{n}\right) \frac{\mathfrak{h}^{\prime \prime}(x)}{2} \\
& \cdot\left(\frac{3 a_{0} \alpha^{2}+3 a_{0} \alpha+6 a_{1} \alpha+a_{0}+6 a_{1}+3 a_{2}}{3 a_{0}(n+\beta)^{2}}-\frac{2 a_{0} b_{0} \alpha(n+\beta)-2 a_{0} b_{1} n(\alpha+1)+a_{0} b_{0}(n+\beta)+2 a_{1} b_{0}(n+\beta)-2 a_{1} b_{1} n}{a_{0} b_{0}(n+\beta)^{2}} x\right. \\
& \left.+\frac{b_{0} n^{2}+2 b_{0} n \beta+b_{0} \beta^{2}-2 b_{1} n^{2}-2 b_{1} n \beta+b_{2} n^{2}}{b_{0}(n+\beta)^{2}} x^{2}\right)+\left(1+x_{n}\right) K_{n}^{\alpha, \beta}\left(\Phi^{2} \psi(y-x) ; x\right) .
\end{aligned}
$$

This yields

$$
\begin{aligned}
(n+\beta)^{2} N_{n}(\mathfrak{h})= & \left(1+x_{n}\right)(n+\beta)^{2} \mathfrak{h}(x)+\left(1+x_{n}\right) \mathfrak{h}^{\prime}(x)(n+\beta)^{2}\left(\frac{2 a_{0} \alpha+a_{0}+2 a_{1}}{2 a_{0}(n+\beta)}-\frac{\left(b_{0} n+b_{0} \beta-b_{1} n\right)}{b_{0}(n+\beta)} x\right)+\left(1+x_{n}\right) \frac{\mathfrak{h}^{\prime \prime}(x)}{2}(n+\beta)^{2} \\
& \left(\frac{3 a_{0} \alpha^{2}+3 a_{0} \alpha+6 a_{1} \alpha+a_{0}+6 a_{1}+3 a_{2}}{3 a_{0}(n+\beta)^{2}}-\frac{2 a_{0} b_{0} \alpha(n+\beta)-2 a_{0} b_{1} n(\alpha+1)+a_{0} b_{0}(n+\beta)+2 a_{1} b_{0}(n+\beta)-2 a_{1} b_{1} n}{a_{0} b_{0}(n+\beta)^{2}} x\right. \\
& \left.+\frac{b_{0} n^{2}+2 b_{0} n \beta+b_{0} \beta^{2}-2 b_{1} n^{2}-2 b_{1} n \beta+b_{2} n^{2}}{b_{0}(n+\beta)^{2}} x^{2}\right)+\left(1+x_{n}\right)(n+\beta)^{2} K_{n}^{\alpha, \beta}\left(\Phi^{2} \psi(y-x) ; x\right)
\end{aligned}
$$

Therefore,

$$
\begin{aligned}
\mid(n+ & \beta)^{2}\left[N_{n}(\mathfrak{h} ; x)-\mathfrak{h}(x)-\mathfrak{h}^{\prime}(x)\left[\frac{2 a_{0} \alpha+a_{0}+2 a_{1}}{2 a_{0}(n+\beta)}-\frac{\left(b_{0} n+b_{0} \beta-b_{1} n\right)}{b_{0}(n+\beta)} x\right]\right. \\
& -\frac{\mathfrak{h}^{\prime \prime}(x)}{2}\left(-\frac{2 a_{0} b_{0} \alpha(n+\beta)-2 a_{0} b_{1} n(\alpha+1)+a_{0} b_{0}(n+\beta)+2 a_{1} b_{0}(n+\beta)-2 a_{1} b_{1} n}{a_{0} b_{0}(n+\beta)^{2}} x\right. \\
& \left.\left.\left.+\frac{b_{0} n^{2}+2 b_{0} n \beta+b_{0} \beta^{2}-2 b_{1} n^{2}-2 b_{1} n \beta+b_{2} n^{2}}{b_{0}(n+\beta)^{2}} x^{2}\right)\right]-\frac{\mathfrak{h}^{\prime \prime}(x)}{2} \cdot \frac{3 a_{0} \alpha^{2}+3 a_{0} \alpha+6 a_{1} \alpha+a_{0}+6 a_{1}+3 a_{2}}{3 a_{0}(n+\beta)^{2}}\right] \\
\leq & M x_{n}(n+\beta)^{2}+M_{1} x_{n}\left[\frac{2 a_{0} \alpha+a_{0}+2 a_{1}}{2 a_{0}(n+\beta)}-\frac{\left(b_{0} n+b_{0} \beta-b_{1} n\right)}{b_{0}(n+\beta)} x\right]+M_{2} \\
& \cdot x_{n}\left(\frac{3 a_{0} \alpha^{2}+3 a_{0} \alpha+6 a_{1} \alpha+a_{0}+6 a_{1}+3 a_{2}}{3 a_{0}(n+\beta)^{2}}-\frac{2 a_{0} b_{0} \alpha(n+\beta)-2 a_{0} b_{1} n(\alpha+1)+a_{0} b_{0}(n+\beta)+2 a_{1} b_{0}(n+\beta)-2 a_{1} b_{1} n}{a_{0} b_{0}(n+\beta)^{2}} x\right. \\
& \left.+\frac{b_{0} n^{2}+2 b_{0} n \beta+b_{0} \beta^{2}-2 b_{1} n^{2}-2 b_{1} n \beta+b_{2} n^{2}}{b_{0}(n+\beta)^{2}} x^{2}\right)+(n+\beta)^{2} K_{n}^{\alpha, \beta}\left((y-x)^{2} \psi(y-x) ; x\right) \\
& +x_{n}\left((n+\beta)^{2} K_{n}^{\alpha, \beta}(y-x)^{2} \psi(y-x) ; x\right),
\end{aligned}
$$


where $M=\sup _{x \in[0, M]}|\mathfrak{h}(x)|, \quad M_{1}=\sup _{x \in[0, M]}\left|\mathfrak{h}^{\prime}(x)\right|$, and $M_{2}$ $=\sup _{x \in[0, M]}\left|\mathfrak{h}^{\prime \prime}(x)\right|$.

Now, we have to prove that

$$
\lim _{n \rightarrow \infty}(n+\beta)^{2} K_{n}^{\alpha, \beta}\left((y-x)^{2} \psi(y-x) ; x\right)=0 .
$$

By applying the Cauchy-Schwartz inequality, we obtain

$$
\begin{aligned}
& (n+\beta)^{2} K_{n}^{\alpha, \beta}\left((y-x)^{2} \psi(y-x) ; x\right) \\
& \quad \leq\left[(n+\beta)^{4} K_{n}^{\alpha, \beta}\left((y-x)^{4} ; x\right)\right]^{1 / 2} \cdot\left[K_{n}^{\alpha, \beta}\left(\psi^{2} ; x\right)\right]^{1 / 2} .
\end{aligned}
$$

Also, by setting $\eta_{x}(y)=(\psi(y-x))^{2}$, we have that $\eta_{x}(x)=0$ and $\eta_{x}(\cdot) \in C[0, M]$. So

$$
K_{n}^{\alpha, \beta}\left(\eta_{x}\right) \longrightarrow 0\left(s t_{A}\right) \quad \text { on } \quad[0, M]
$$

Now, from the last relation, (74), (75), and Lemma 20, we obtain that

$$
(n+\beta)^{2} K_{n}^{\alpha, \beta}\left((y-x)^{2} \psi(y-x) ; x\right) \longrightarrow 0\left(s t_{A}\right) \quad \text { on } \quad[0, M]
$$

From the construction of $\left(x_{n}\right)$, it follows that $(n+\beta)^{2}$ $x_{n} \longrightarrow 0\left(s t_{A}\right)$ on $[0, M]$.

For a given $\varepsilon>0$, we define the following sets:

$$
\begin{aligned}
A= & \left\{n: \mid(n+\beta)^{2}\left[N_{n}(\mathfrak{h} ; x)-\mathfrak{h}(x)-\mathfrak{h}^{\prime}(x)\left[\frac{2 a_{0} \alpha+a_{0}+2 a_{1}}{2 a_{0}(n+\beta)}-\frac{\left(b_{0} n+b_{0} \beta-b_{1} n\right)}{b_{0}(n+\beta)} x\right]\right.\right. \\
& -\frac{\mathfrak{h}^{\prime \prime}(x)}{2}\left(-\frac{2 a_{0} b_{0} \alpha(n+\beta)-2 a_{0} b_{1} n(\alpha+1)+a_{0} b_{0}(n+\beta)+2 a_{1} b_{0}(n+\beta)-2 a_{1} b_{1} n}{a_{0} b_{0}(n+\beta)^{2}} x\right. \\
& \left.\left.\left.+\frac{b_{0} n^{2}+2 b_{0} n \beta+b_{0} \beta^{2}-2 b_{1} n^{2}-2 b_{1} n \beta+b_{2} n^{2}}{b_{0}(n+\beta)^{2}} x^{2}\right)\right]-\frac{\mathfrak{h}^{\prime \prime}(x)}{2} \cdot \frac{3 a_{0} \alpha^{2}+3 a_{0} \alpha+6 a_{1} \alpha+a_{0}+6 a_{1}+3 a_{2}}{3 a_{0}(n+\beta)^{2}} \mid \geq \varepsilon\right\},
\end{aligned}
$$

$A_{1}=\left|\left\{n:\left|x_{n}(n+\beta)^{2}\right| \geq \frac{\varepsilon}{5 M}\right\}\right|$,

$A_{2}=\left|\left\{n:\left|x_{n}\left[\frac{2 a_{0} \alpha+a_{0}+2 a_{1}}{2 a_{0}(n+\beta)}-\frac{\left(b_{0} n+b_{0} \beta-b_{1} n\right)}{b_{0}(n+\beta)} x\right]\right| \geq \frac{\varepsilon}{5 M_{1}}\right\}\right|$,

$A_{3}=\mid\left\{n: \mid x_{n}\left(\frac{3 a_{0} \alpha^{2}+3 a_{0} \alpha+6 a_{1} \alpha+a_{0}+6 a_{1}+3 a_{2}}{3 a_{0}(n+\beta)^{2}}-\frac{2 a_{0} b_{0} \alpha(n+\beta)-2 a_{0} b_{1} n(\alpha+1)+a_{0} b_{0}(n+\beta)+2 a_{1} b_{0}(n+\beta)-2 a_{1} b_{1} n}{a_{0} b_{0}(n+\beta)^{2}} x\right.\right.$

$$
\left.\left.+\frac{b_{0} n^{2}+2 b_{0} n \beta+b_{0} \beta^{2}-2 b_{1} n^{2}-2 b_{1} n \beta+b_{2} n^{2}}{b_{0}(n+\beta)^{2}} x^{2}\right) \mid \geq \frac{\varepsilon}{5 M_{2}}\right\} \mid
$$

$A_{4}=\left|\left\{n:\left|(n+\beta)^{2} K_{n}^{\alpha, \beta}\left((y-x)^{2} \psi(y-x) ; x\right)\right| \geq \frac{\varepsilon}{5}\right\}\right|$,

$A_{5}=\left|\left\{n:\left|x_{n}(n+\beta)^{2} K_{n}^{\alpha, \beta}\left((y-x)^{2} \psi(y-x) ; x\right)\right| \geq \frac{\varepsilon}{5}\right\}\right|$.

From last relations, we obtain that $A \leq A_{1}+A_{2}+A_{3}+$ $A_{4}+A_{5}$. Hence, the result follows.

Remark 22. We see that the operators $\left(N_{n}\right)$ (see Example 13) do not satisfy a Voronovskaya-type theorem in the usual sense.

\section{Grüss-Voronovskaya-Type Theorems}

This kind of result, for the first time, was shown in [30].
Theorem 23 (see [31]). Let $E=\{\mathfrak{h}: x \in[0, \infty),(\mathfrak{h}(x) /(1+$ $\left.x^{2}\right)$ ), is convergent as $\left.x \longrightarrow \infty\right\}$ and

$$
\lim _{n \rightarrow \infty} \frac{B^{(m)}(n x)}{B(n x)}=1 \quad \text { for } \quad m=1,2 .
$$

If $\mathfrak{h} \in C[0, \infty) \cap E$, then

$$
\lim _{n \rightarrow \infty} K_{n}^{\alpha, \beta}(f, x)=f(x),
$$


and the operators $K_{n}^{\alpha, \beta}(\mathfrak{h}, x)$ converge uniformly in each compact subset of $[0, \infty)$.

Now, we are ready to prove the following result.
Theorem 24. For $\mathfrak{h}, \mathfrak{h}^{\prime}, \mathfrak{h}^{\prime \prime} \in C[0, \infty)$ and any $x \in[0, \infty)$, $\lim _{n \rightarrow \infty} B^{(i)}(n x) / B(n x)=1$, for $i \in\{1,2,3,4,5,6\}$, where $B^{(i)}$ denotes the ith derivative of $B$. Then,

$$
\begin{aligned}
\mid(n+ & \beta)\left(K_{n}^{\alpha, \beta}(\mathfrak{h}, x)-\mathfrak{h}(x)\right)-\mathfrak{h}^{\prime}(x)\left(\frac{2 a_{0} \alpha+a_{0}+2 a_{1}}{2 a_{0}(n+\beta)}-\frac{\left(b_{0} n+b_{0} \beta-b_{1} n\right)}{b_{0}(n+\beta)} x\right)-(n+\beta) \frac{\mathfrak{h}^{\prime \prime}(x)}{2} \\
& \cdot\left[\frac{3 a_{0} \alpha^{2}+3 a_{0} \alpha+6 a_{1} \alpha+a_{0}+6 a_{1}+3 a_{2}}{3 a_{0}(n+\beta)^{2}}-\frac{2 a_{0} b_{0} \alpha(n+\beta)-2 a_{0} b_{1} n(\alpha+1)+a_{0} b_{0}(n+\beta)+2 a_{1} b_{0}(n+\beta)-2 a_{1} b_{1} n}{a_{0} b_{0}(n+\beta)^{2}} x\right. \\
& \left.+\frac{b_{0} n^{2}+2 b_{0} n \beta+b_{0} \beta^{2}-2 b_{1} n^{2}-2 b_{1} n \beta+b_{2} n^{2}}{b_{0}(n+\beta)^{2}} x^{2}\right] \mid=0(1) \omega\left(\mathfrak{h}^{\prime \prime}, n^{-1 / 2}\right),
\end{aligned}
$$

as $n \longrightarrow \infty$.

Proof. From Taylor's theorem, we have

$$
\mathfrak{h}(u)=\mathfrak{h}(x)+\mathfrak{h}^{\prime}(x)(u-x)+\frac{\mathfrak{h}^{\prime \prime}(x)}{2}(u-x)^{2}+R(u, x),
$$

where $R(u, x)=\left(\left(\mathfrak{h}^{\prime \prime}(\theta)-\mathfrak{h}^{\prime \prime}(x)\right) / 2\right)(u-x)^{2}$, for $\theta \in(u, x)$. Now, we obtain

$$
\begin{aligned}
& \mid K_{n}^{\alpha, \beta}(\mathfrak{h}, x)-\mathfrak{h}(x)-\mathfrak{h}^{\prime}(x) K_{n}^{\alpha, \beta}((u-x) ; x) \\
& \quad-\frac{\mathfrak{h}^{\prime \prime}(x)}{2} K_{n}^{\alpha, \beta}\left((u-x)^{2} ; x\right) \mid \leq K_{n}^{\alpha, \beta}(|R(u, x)|, x) .
\end{aligned}
$$

From which we get that

$$
\begin{aligned}
\mid(n+\beta) & \left(K_{n}^{\alpha, \beta}(\mathfrak{h}, x)-\mathfrak{h}(x)\right)-\mathfrak{h}^{\prime}(x)\left(\frac{2 a_{0} \alpha+a_{0}+2 a_{1}}{2 a_{0}(n+\beta)}-\frac{\left(b_{0} n+b_{0} \beta-b_{1} n\right)}{b_{0}(n+\beta)} x\right)-(n+\beta) \frac{\mathfrak{h}^{\prime \prime}(x)}{2} \\
\cdot & {\left[\frac{3 a_{0} \alpha^{2}+3 a_{0} \alpha+6 a_{1} \alpha+a_{0}+6 a_{1}+3 a_{2}}{3 a_{0}(n+\beta)^{2}}-\frac{2 a_{0} b_{0} \alpha(n+\beta)-2 a_{0} b_{1} n(\alpha+1)+a_{0} b_{0}(n+\beta)+2 a_{1} b_{0}(n+\beta)-2 a_{1} b_{1} n}{a_{0} b_{0}(n+\beta)^{2}} x\right.} \\
& \left.+\frac{b_{0} n^{2}+2 b_{0} n \beta+b_{0} \beta^{2}-2 b_{1} n^{2}-2 b_{1} n \beta+b_{2} n^{2}}{b_{0}(n+\beta)^{2}} x^{2}\right] \mid \leq(n+\beta) \cdot K_{n}^{\alpha, \beta}(|R(u, x)|, x) .
\end{aligned}
$$
have

By the properties of modulus of continuity modulus, we

$$
\left|\frac{\mathfrak{h}^{\prime \prime}(\theta)-\mathfrak{h}^{\prime \prime}(x)}{2 !}\right| \leq \frac{1}{2 !}\left(1+\frac{|\theta-x|}{\delta}\right) \omega\left(\mathfrak{h}^{\prime \prime}, \delta\right) .
$$

On the other hand,

$$
\left|\frac{\mathfrak{h}^{\prime \prime}(\theta)-\mathfrak{h}^{\prime \prime}(x)}{2 !}\right| \leq \begin{cases}\omega\left(\mathfrak{h}^{\prime \prime}, \delta\right), & |u-x| \leq \delta, \\ \frac{(t-x)^{4}}{\delta^{4}} \omega\left(\mathfrak{h}^{\prime \prime}, \delta\right), & |u-x| \geq \delta .\end{cases}
$$

For $0<\delta<1$, we obtain that

$$
\left|\frac{\mathfrak{h}^{\prime \prime}(\theta)-\mathfrak{h}^{\prime \prime}(x)}{2 !}\right| \leq \omega\left(\mathfrak{h}^{\prime^{\prime \prime}}, \delta\right)\left(1+\frac{(u-x)^{4}}{\delta^{4}}\right)
$$

which gives

$$
\begin{aligned}
|R(u, x)| & \leq \omega\left(\mathfrak{h}^{\prime \prime}, \delta\right)\left(1+\frac{(u-x)^{4}}{\delta^{4}}\right)(u-x)^{2} \\
& =\omega\left(\mathfrak{h}^{\prime \prime}, \delta\right)\left((u-x)^{2}+\frac{(u-x)^{6}}{\delta^{4}}\right) .
\end{aligned}
$$


By the linearity of $K_{n}^{\alpha, \beta}$ and the above relation, we obtain

$$
\begin{aligned}
& K_{n}^{\alpha, \beta}(|R(u, x)|, x) \\
& \quad \leq \omega\left(\mathfrak{h}^{\prime \prime}, \delta\right)\left(K_{n}^{\alpha, \beta}\left((u-x)^{2}, x\right)+\frac{1}{\delta^{4}} K_{n}^{\alpha, \beta}\left((u-x)^{6}, x\right)\right) .
\end{aligned}
$$

Taking into consideration Proposition 9, we have

$$
\begin{aligned}
K_{n}^{\alpha, \beta}(|R(u, x)|, x) & \leq \omega\left(\mathfrak{h}^{\prime \prime}, \delta\right)\left(0\left(\frac{1}{n}\right)+\frac{1}{\delta^{4}} 0\left(\frac{1}{n^{3}}\right)\right) \\
& =0\left(\frac{1}{n}\right) \omega\left(\mathfrak{h}^{\prime \prime}, \delta\right) .
\end{aligned}
$$

For $\delta=n^{-1 / 2}$, we complete the proof.

Theorem 25. Let $\mathfrak{h}^{\prime}(x), g^{\prime}(x), \mathfrak{h}^{\prime \prime}(x), g^{\prime \prime}(x),(\mathfrak{h} g)^{\prime}(x)$, $(\mathfrak{h} g)^{\prime \prime}(x) \in C[0, \infty) \cap E$, and

$$
\lim _{n \rightarrow \infty} \frac{B^{(m)}(n x)}{B(n x)}=1 \quad \text { for } \quad m=0,1,2, \cdots, 6, \quad x \in[0, M],
$$

where $B^{(m)}$ is the $m$ th derivative of $B$. Then,

$$
\begin{aligned}
\lim _{n \rightarrow \infty}(n+\beta)\left[K_{n}^{\alpha, \beta}(\mathfrak{h} g, x)-K_{n}^{\alpha, \beta}(\mathfrak{h}, x) \cdot K_{n}^{\alpha, \beta}(g, x)\right] \\
\quad=x \mathfrak{h}^{\prime}(x) g^{\prime}(x) .
\end{aligned}
$$

Proof. We know that

$$
\begin{aligned}
(n+\beta) & \left\{K_{n}^{\alpha, \beta}(\mathfrak{h} g, x)-K_{n}^{\alpha, \beta}(\mathfrak{h}, x) K_{n}^{\alpha, \beta}(g, x)\right\} \\
= & (n+\beta)\left\{K_{n}^{\alpha, \beta}(\mathfrak{h} g, x)-(\mathfrak{h} g)(x)\right. \\
& -(\mathfrak{h} g)^{\prime}(x) K_{n}^{\alpha, \beta}((u-x), x)-\frac{(\mathfrak{h} g)^{\prime \prime}(x)}{2} K_{n}^{\alpha, \beta}\left((u-x)^{2}, x\right) \\
& -g(x)\left[K_{n}^{\alpha, \beta}(\mathfrak{h}, x)-\mathfrak{h}(x)-\mathfrak{h}^{\prime}(x) K_{n}^{\alpha, \beta}((u-x), x)\right. \\
& \left.-\frac{\mathfrak{h}^{\prime \prime}(x)}{2} K_{n}^{\alpha, \beta}\left((u-x)^{2}, x\right)\right] \\
& -K_{n}^{\alpha, \beta}(\mathfrak{h}, x)\left[K_{n}^{\alpha, \beta}(g, x)-g(x)-g^{\prime}(x) K_{n}^{\alpha, \beta}((u-x), x)\right. \\
& \left.-\frac{g^{\prime \prime}(x)}{2} K_{n}^{\alpha, \beta}\left((u-x)^{2}, x\right)\right] \\
& +\frac{g^{\prime \prime}(x)}{2} K_{n}^{\alpha, \beta}\left((u-x)^{2}, x\right)\left[\mathfrak{h}(x)-K_{n}^{\alpha, \beta}(\mathfrak{h}, x)\right] \\
& +\mathfrak{h}^{\prime}(x) g^{\prime}(x) K_{n}^{\alpha, \beta}\left((u-x)^{2}, x\right)-g^{\prime}(x) K_{n}^{\alpha, \beta}((u-x), x) \\
& \left.\cdot\left[K_{n}^{\alpha, \beta}(\mathfrak{h}, x)-\mathfrak{h}(x)\right]\right\} . \\
&
\end{aligned}
$$

From Proposition 9 and Theorems 23 and 24, we obtain

$$
\begin{aligned}
\lim _{n \rightarrow \infty}(n+\beta)\left\{K_{n}^{\alpha, \beta}(\mathfrak{h} g, x)-K_{n}^{\alpha, \beta}(\mathfrak{h}, x) K_{n}^{\alpha, \beta}(g, x)\right\} \\
\quad=x \mathfrak{h}^{\prime}(x) g^{\prime}(x) .
\end{aligned}
$$

\section{Data Availability}

No data were used to support this study.

\section{Conflicts of Interest}

The authors declare that there are no conflicts of interest regarding the publication of this paper.

\section{References}

[1] J. A. Fridy and H. I. Miller, "A matrix characterization of statistical convergence,” Analysis, vol. 11, no. 1, pp. 59-66, 1991.

[2] F. Basar, Summability Theory and Its Applications, Bentham Science Publishers, Ltd., Oak Park, IL, USA, 2011.

[3] N. L. Braha, "Some weighted equi-statistical convergence and Korovkin type-theorem," Results in Mathematics, vol. 70, no. 3-4, pp. 433-446, 2016.

[4] N. L. Braha, V. Loku, and H. M. Srivastava, " $\Lambda^{2}$-weighted statistical convergence and Korovkin and Voronovskaya type theorems," Applied Mathematics and Computation, vol. 266, pp. 675-686, 2015.

[5] N. L. Braha, H. M. Srivastava, and S. A. Mohiuddine, "A Korovkin's type approximation theorem for periodic functions via the statistical summability of the generalized de la Vallée Poussin mean," Applied Mathematics and Computation, vol. 228, pp. 162-169, 2014.

[6] N. L. Braha, "Some properties of new modified SzászMirakyan operators in polynomial weight spaces via power summability method," Bulletin of Mathematical Analysis and Applications, vol. 10, no. 3, pp. 53-65, 2018.

[7] N. L. Braha, "Some properties of Baskakov-Schurer-Szász operators via power summability methods," Quaestiones Mathematicae, vol. 42, no. 10, pp. 1411-1426, 2019.

[8] U. Kadak, N. L. Braha, and H. M. Srivastava, "Statistical weighted $\mathscr{B}$-summability and its applications to approximation theorems," Applied Mathematics and Computation, vol. 302, pp. 80-96, 2017.

[9] M. Mursaleen, V. Karakaya, M. Erturk, and F. Gursoy, "Weighted statistical convergence and its application to Korovkin type approximation theorem," Applied Mathematics and Computation, vol. 218, no. 18, pp. 9132-9137, 2012.

[10] E. Tas and T. Yurdakadim, "Approximation by positive linear operators in modular spaces by power series method," Positivity, vol. 21, no. 4, pp. 1293-1306, 2017.

[11] W. Kratz and U. Stadtmuller, "Tauberian theorems for $J_{p}$ -summability," Journal of Mathematical Analysis and Applications, vol. 139, no. 2, pp. 362-371, 1989.

[12] U. Stadtmuller and A. Tali, "On certain families of generalized Nörlund methods and power series methods," Journal of Mathematical Analysis and Applications, vol. 238, no. 1, pp. 44-66, 1999.

[13] S. Varma, S. Sucu, and G. Icoz, "Generalization of Szasz operators involving Brenke type polynomials," Computers \& Mathematcs with Applications, vol. 64, no. 2, pp. 121-127, 2012. 
[14] J. Boos, Classical and Modern Methods in Summability, Oxford University Press, Oxford, 2000.

[15] M. Ahasan and M. Mursaleen, "Generalized Szász-Mirakjan type operators via q-calculus and approximation properties," Applied Mathematics and Computation, vol. 371, article 124916, 2020.

[16] K. J. Ansari, M. Mursaleen, K. P. M. Shareef, and M. Ghouse, "Approximation by modified Kantorovich-Szász type operators involving Charlier polynomials," Advances in Difference Equations, vol. 2020, no. 1, 2020.

[17] M. Mursaleen, A. Naaz, and A. Khan, "Improved approximation and error estimations by King type $(p, q)$-Szasz- Mirakjan Kantorovich operators," Applied Mathematics and Computation, vol. 348, pp. 175-185, 2019.

[18] M. Mursaleen and S. Rahman, "Dunkl generalization of qSzász-Mirakjan operators which preserve $x^{2}$," Filomat, vol. 32, no. 3, pp. 733-747, 2018.

[19] M. Nasiruzzaman, A. Mukheimer, and M. Mursaleen, “A Dunkl-Type generalization of Szász-Kantorovich operators via post-quantum calculus," Symmetry, vol. 11, no. 2, p. 232, 2019.

[20] M. Mursaleen and K. J. Ansari, "On Chlodowsky variant of Szász operators by Brenke type polynomials," Applied Mathematics and Computation, vol. 271, pp. 991-1003, 2015.

[21] T. Mansour and M. Schork, Commutation Relations, Normal Ordering and Stirling Numbers, Chapman \& Hall/CRC an imprint of Taylor \& Francis LLC, 2015.

[22] O. G. Atlihan, M. Unver, and O. Duman, "Korovkin theorems on weighted spaces: revisited," Periodica Mathematica Hungarica, vol. 75, no. 2, pp. 201-209, 2017.

[23] M. Campiti and G. Metafune, " $L^{p}$-convergence of BernsteinKantorovich-type operators," Annales Polonici Mathematici, vol. 63, no. 3, pp. 273-280, 1996.

[24] O. Duman, M. K. Khan, and C. Orhan, "A-Statistical convergence of approximating operators," Mathematical Inequalities \& Applications, vol. 6, pp. 689-699, 1998.

[25] M. Kirisci and A. Karaisa, "Fibonacci statistical convergence and Korovkin type approximation theorems," Journal of Inequalities and Applications, vol. 2017, no. 1, Article ID 229, 2017.

[26] V. Loku and N. L. Braha, "Some weighted statistical convergence and Korovkin type-theorem," Journal of Inequalities and Special Functions, vol. 8, no. 3, pp. 139-150, 2017.

[27] D. Soylemez and M. Unver, "Korovkin type theorems for Cheney-Sharma operators via summability methods," Results in Mathematics, vol. 72, no. 3, pp. 1601-1612, 2017.

[28] T. A. Ş. Emre, "Some results concerning Mastroianni operators by power series method," Communications Faculty Of Science University of Ankara Series, vol. 65, no. 1, pp. 187-195, 2016.

[29] M. Unver, "Abel transforms of positive linear operators," AIP Conference Proceedings, vol. 1558, pp. 1148-1151, 2013.

[30] S. G. Gal and H. Gonska, "Grüss and Grüss-Voronovskayatype estimates for some Bernstein-type polynomials of real and complex variables," Jaen Journal on Approximation, vol. 7, no. 1, pp. 97-122, 2015.

[31] R. Aktas, B. Çekim, and F. Tasdelen, “A Kantorovich-Stancu type generalization of Szasz operators including Brenke type polynomials," Journal of Function Spaces and Applications, vol. 2013, article 935430, pp. 1-9, 2013. 